\title{
Quantum mechanics in non(anti)commutative superspace
}

\author{
Leon G. Aldrovandi and Fidel A. Schaposnik* \\ Departamento de Física, Universidad Nacional de La Plata \\ C.C. 67, (1900) La Plata, Argentina \\ E-mail: aldrovandi@fisica.unlp.edu.ar, fidel@fisica.unlp.edu.ar
}

Abstract: We consider non(anti)commutative (NAC) deformations of $d=1 \mathcal{N}=2$ superspace. We find that, in the chiral base, the deformation preserves only a half of the original (linearly realized) supercharge algebra, as it usually happens in NAC field theories. We obtain in terms of a real supermultiplet a closed expression for a deformed Quantum Mechanics Lagrangian in which the original superpotential is smeared, similarly to what happens for the two dimensional deformed sigma model. Quite unexpectedly, we find that a second conserved charge can be constructed which leads to a nonlinear field realization of the supersymmetry algebra, so that finally the deformed theory has as many conserved supercharges as the undeformed one. The quantum behavior of these supercharges is analyzed.

Keywords: Superspaces, Non-Commutative Geometry, Field Theories in Lower Dimensions, Supersymmetry Breaking.

\footnotetext{
${ }^{*}$ F.A.S. is associated with CICBA.
} 


\section{Contents}

1. Introduction 1

2. $\mathcal{N}=2$ deformed superspace $\quad 2$

3. Lagrangian and classical supersymmetries 4

$\begin{array}{ll}\text { 4. Quantum supersymmetries } & 7\end{array}$

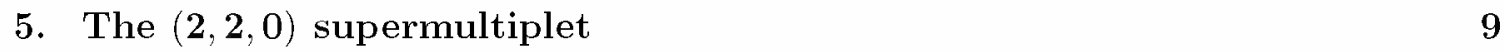

6. Summary and discussion 10

\section{Introduction}

New aspects of supersymmetric field theories have been recently analyzed by deforming superspace so that both bosonic and fermionic coordinates obey non-trivial commutation and anticommutation relations. Such deformed superspaces, first proposed in [1]-[8], are known as non(anti)commutative (NAC) superspaces and they have recently attracted much attention both in connection with string theory [9]-[14] and also with field theories, like in studies of modified BPS solutions, anomalies and condensates in SUSY gluodynamics, etc $[15]-[18]$.

Supersymmetric Quantum Mechanics (SUSY QM) [19]-[20] provides a simple and, at the same time, quite adequate framework for understanding SUSY breaking non-perturbatively. In particular, after the introduction of the Witten index [20], many interesting topological aspects of SUSY QM were clarified shedding light into more involved problem of SUSY breaking in quantum field theory. For instance, the connection between the Witten index and QM yields to a beautiful derivation of the Atiyah-Singer index theorem [21]-[22], relevant for analysis of SUSY field theories.

In this work we study the NAC deformation of $d=1 \mathcal{N}=2$ superspace and the possibility of formulating Quantum Mechanics in such a superspace. Our purpose is twofold: on the one hand we intend to fill a gap in the study of NAC theories by analyzing the one dimensional case. We think that this is interesting by its own and for possible applications in condensed matter problems. On the other hand, as the study of QM is simpler than that of field theories, we shall use it to analyze an important issue of NAC theories, namely the question of how many supercharges are effectively broken by the deformation. We know that in NAC field theories, once the deformation is implemented, only a fraction (usually a half) of the original linearly realized algebra is left. Remarkably, we shall see that in 
the Quantum Mechanics case a new, deformed supercharge, can be constructed (at least classically), which corresponds to a non-linear realization of the supersymmetry algebra. This implies that the deformed theory finally has as many conserved supercharges as the undeformed one.

The paper is organized as follows. In section II we present our definition of the NAC superspace and, working in the chiral base, we show that the deformation preserves only half of the supercharge algebra. Then, in section III we construct a deformed QM Lagrangian by replacing the usual product between superfields by the Moyal-Weyl product. Adapting results obtained in [23]-[25] for the $d=2, \mathcal{N}=2$ NAC sigma-model, we find a $d=1$ Lagrangian which, written in components, takes the same form as the undeformed one but with an effective superpotential that depends not only on a scalar but also on the auxiliary field. We then present two charges, conserved in the deformed model, which lead to a nonlinear field realization of the $\mathcal{N}=2$ superalgebra. In section IV we study quantum aspects of the deformed theory. Using a path-integral version of the Witten index $\mathcal{W}$ we show that whenever the superpotential is such that SUSY is not broken in the undeformed model, $\mathcal{W}$ remains unchanged by the deformation and then the difference between the number of boson and fermion states annihilated by the supercharges can be determined. Section $\mathrm{V}$ is devoted to the formulation of the deformed theory using chiral supermultiplets, this avoiding the use of auxiliary fields. However, in order to preserve the chirality condition the class of deformation turns to be restricted in a way such that the resulting action coincides with the undeformed one. We present in section VI a summary and discussion of our results.

\section{2. $\mathcal{N}=2$ deformed superspace}

In the superspace formulation of supersymmetric Quantum Mechanics, apart from the (commuting) real time variable $\tau$, one considers anticommuting (Grassmann) variables $\theta$ and its complex conjugated $\bar{\theta}$. A deformed superspace can be then defined by modifying the anticommutation relations among the $\theta$ and $\bar{\theta}$ variables and even their commutation relations with $\tau$.

In the ordinary case, the $\mathcal{N}=2, d=1$ superspace $\mathbb{R}^{(1 / 2)}$ can be parameterized by the coordinates

$$
\mathbb{R}^{(1 \mid 2)}=(\tau, \theta, \bar{\theta}), \quad \tau \in \mathbb{R},(\theta)^{\dagger}=\bar{\theta} .
$$

The most general deformation of the fermionic sector of this superspace is

$$
\{\theta, \theta\}=C, \quad\{\bar{\theta}, \bar{\theta}\}=\bar{C}, \quad\{\theta, \bar{\theta}\}=\widehat{C}
$$

where $C, C$ and $\widehat{C}$ are c-numbers having dimensions $\mathrm{m}^{-1}$ since we take $[\theta]=[\bar{\theta}]=\mathrm{m}^{-1 / 2}$. Concerning the commutation rules for the time variable with $\theta$ and $\bar{\theta}$, we postpone the choice to the introduction of a "chiral" time variable (see below).

As in the case of space-time deformations, a $d=1$ theory in the non(anti)commutative superspace defined by (2.2) can be realized using ordinary Grassmann coordinates but 
multiplying superfields with an appropriate Moyal-Weyl product. Indeed, given for example scalar superfields of the form

$$
\Phi(\tau, \theta, \bar{\theta})=\phi(\tau)+\theta \psi(\tau)+\bar{\psi}(\tau) \bar{\theta}+\theta \bar{\theta} F(\tau)
$$

with $\phi$ a scalar, $\psi$ a fermion and $F$ an auxiliary field, one can define the Moyal-Weyl product which implements the deformation (2.2) as

$$
\begin{aligned}
& \Phi_{1}(\tau, \theta, \bar{\theta}) * \Phi_{2}(\tau, \theta, \bar{\theta})=\Phi_{1} \exp \left(-\frac{C}{2} \frac{\overleftarrow{\partial}}{\partial \theta} \frac{\vec{\partial}}{\partial \theta}-\frac{\widehat{C}}{2} \frac{\overleftarrow{\partial}}{\partial \theta} \frac{\vec{\partial}}{\partial \theta}-\frac{\widehat{C}}{2} \frac{\grave{\partial}}{\partial \theta} \frac{\vec{\partial}}{\partial \theta}-\frac{\vec{C}}{2} \frac{\overleftarrow{\partial}}{\partial \theta} \frac{\vec{\partial}}{\partial \theta}\right) \Phi_{2} \\
& =\Phi_{1}\left[1-\frac{C}{2} \frac{\overleftarrow{\partial}}{\partial \theta} \frac{\vec{\partial}}{\partial \theta}-\frac{\widehat{C}}{2} \frac{\grave{\partial}}{\partial \theta} \frac{\vec{\partial}}{\partial \theta}-\frac{\widehat{C}}{2} \frac{\grave{\partial}}{\partial \theta} \frac{\vec{\partial}}{\partial \theta}-\frac{C}{2} \frac{\overleftarrow{\partial}}{\partial \theta} \frac{\vec{\partial}}{\partial \theta}-\frac{1}{4} r^{2} \frac{\overleftarrow{\partial}}{\partial \theta} \frac{\grave{\partial}}{\partial \theta} \frac{\vec{\partial}}{\partial \theta} \frac{\vec{\partial}}{\partial \theta}\right] \Phi_{2}
\end{aligned}
$$

with $c^{2}=C \bar{C}-\widehat{C} \widehat{C}$ and $\Phi \frac{\bar{\partial}}{\partial \theta}=(-1)^{f|\Phi| \frac{\partial \Phi}{\partial \theta}}$, where $f[\Phi]$ is the Grassman character of the superfield $\Phi$.

In the undeformed case, the differential operators $Q$ and $\bar{Q}$ generating the supersymmetry transformations are given by

$$
Q=\frac{\partial}{\partial \theta}+i \bar{\theta} \frac{\partial}{\partial \tau}, \quad \bar{Q}=-\frac{\partial}{\partial \bar{\theta}}-i \theta \frac{\partial}{\partial \tau} .
$$

and satisfy the undeformed SUSY $\mathcal{N}=2$ QM algebra

$$
\{Q, Q\}=0, \quad\{\bar{Q}, \bar{Q}\}=0, \quad\{Q, \bar{Q}\}=-2 i \frac{\partial}{\partial \tau} .
$$

It is sometimes useful to work with the supercharges realizations

$$
Q=\frac{\partial}{\partial \theta}+i b \bar{\theta} \frac{\partial}{\partial t}, \quad \bar{Q}=-\frac{\partial}{\partial \bar{\theta}}-i \bar{b} \theta \frac{\partial}{\partial t} .
$$

which can be obtained from (2.5) through the change of variables

$$
\tau \rightarrow t=\tau-i(1-b) \theta \bar{\theta}
$$

and satisfy the familiar supersymmetry algebra when $b+\bar{b}=2$. Concerning the covariant derivatives $D$ and $\bar{D}$ anticommuting with the supersymmetry charges, they take the form

$$
D=\frac{\partial}{\partial \theta}-i \bar{b} \bar{\theta} \frac{\partial}{\partial t}, \quad \bar{D}=-\frac{\partial}{\partial \bar{\theta}}+i b \theta \frac{\partial}{\partial t} .
$$

It is worthwhile to stress that we shall always consider the same formal expression for the Moyal-Weyl product (2.4), independently of having changed the temporal variable according to (2.8). This means that after the change $\tau \rightarrow t$, derivatives with respect to $\theta$ and $\bar{\theta}$ in (2.4) should be taken at fixed $t$.

In the deformed case, the algebra of the supercharges $Q$ and $\bar{Q}$ changes to

$$
\begin{aligned}
& \{Q, Q\}=-b^{2} \bar{C} \frac{\partial^{2}}{\partial t^{2}} \\
& \{\bar{Q}, \bar{Q}\}=-\bar{b}^{2} C \frac{\partial^{2}}{\partial t^{2}} \\
& \{Q, \bar{Q}\}=-i(b+\bar{b}) \frac{\partial}{\partial t}+b \bar{b} \widehat{C} \frac{\partial^{2}}{\partial t^{2}}
\end{aligned}
$$


Different choices for $b, b$ and the $C$-parameters make possible to preserve different sectors of the undeformed SUSY algebra. To select suitable values for these parameters, it is convenient to see under which conditions the operators $Q$ and $Q$ still satisfy the Leibnitz rule. When $Q$ and $\bar{Q}$ act on $*$ product of superfields, one has

$$
\begin{aligned}
& Q *\left(\Phi_{1} * \Phi_{2}\right)= \\
& \left(Q * \Phi_{1}\right) * \Phi_{2}+\Phi_{1} *\left(Q * \Phi_{2}\right)+i b\left[\bar{\theta} \frac{\partial}{\partial t}\left(\Phi_{1} * \Phi_{2}\right)-\left(\bar{\theta} \frac{\partial \Phi_{1}}{\partial t}\right) * \Phi_{2}-\Phi_{1} *\left(\bar{\theta} \frac{\partial \Phi_{2}}{\partial t}\right)\right] \\
& \bar{Q} *\left(\Phi_{1} * \Phi_{2}\right)= \\
& \left(\bar{Q} * \Phi_{1}\right) * \Phi_{2}+\Phi_{1} *\left(\bar{Q} * \Phi_{2}\right)-i \bar{b}\left[\theta \frac{\partial}{\partial t}\left(\Phi_{1} * \Phi_{2}\right)-\left(\theta \frac{\partial \Phi_{1}}{\partial t}\right) * \Phi_{2}-\Phi_{1} *\left(\theta \frac{\partial \Phi_{2}}{\partial t}\right)\right]
\end{aligned}
$$

One can see that both supersymmetry generators cannot satisfy simultaneously the Leibnitz rule due to the constraint $b+\bar{b}=2$. From here on we shall work in the chiral base, which corresponds to the $b=0, \bar{b}=2$ case and then, according to (2.10), only the $\mathcal{N}=\frac{2}{2}$ SUSY subalgebra associated to the $Q$ generator will be preserved. Let us also note that this election is consistent with

$$
[t, \theta]=[t, \bar{\theta}]=0
$$

which corresponds, in terms of the original coordinates $(\tau, \theta, \bar{\theta})$, to

$$
[\tau, \tau]=0, \quad[\tau, \theta]=i(\bar{C} \theta-C \bar{\theta}), \quad[\tau, \bar{\theta}]=i(\bar{C} \theta-\bar{C} \bar{\theta})
$$

Finally, the supercharge and covariant derivatives take the form

$$
\begin{array}{ll}
Q=\frac{\partial}{\partial \theta}, & \bar{Q}=-\frac{\partial}{\partial \theta}-2 i \theta \frac{\partial}{\partial t}, \\
D=\frac{\partial}{\partial \theta}-2 i \bar{\theta} \frac{\partial}{\partial t}, & D=-\frac{\partial}{\partial \theta} .
\end{array}
$$

\section{Lagrangian and classical supersymmetries}

There are two basic kinds of $\mathcal{N}=2$ supermultiplets in $d=1$ dimension. To classify them it is convenient to introduce the notation $(\mathbf{m}, \mathbf{n}, \mathbf{n}-\mathbf{m})$ to identify an off-shell $\mathcal{N}=\mathbf{n}, d=1$ supermultiplet with $\mathbf{m}$ physical bosons, $\mathbf{n}$ fermions and $\mathbf{n}-\mathbf{m}$ auxiliary bosonic components. Thus, the $(\mathbf{1}, \mathbf{2}, \mathbf{1})$ supermultiplet, also known as $\mathcal{N}=2 a$ model, is constructed from unconstrained real $\mathcal{N}=2$ superfields while the $(2,2,0)$ supermultiplet or $\mathcal{N}=2 b$ model use complex chiral superfields. In the undeformed superspace, $\mathcal{N}=2 a$ models can be obtained by dimensional reduction of $(1,1)$ supersymmetric two dimensional sigmamodels, while the reduction of $(2,0)$ supersymmetric two dimensional sigma-models leads to $\mathcal{N}=2 b$ models.

A deformed $\mathcal{N}=2$ supersymmetric action constructed by using the $(\mathbf{1}, \mathbf{2}, \mathbf{1})$ supermultiplet takes the form

$$
\left.S=\int d t d \theta d \theta\left[-\frac{1}{2}(D * \Phi) *(\bar{D} * \Phi)-W_{*}(\Phi)\right]\right)
$$


where one defines the deformed superpotential $W_{*}(\Phi)$ from the function $W(\Phi)$ in the underformed case through

$$
W(\Phi)=\sum_{n=0}^{\infty} \frac{1}{n !} W^{(n)} \Phi \Phi \stackrel{(n)}{.} \Phi \longrightarrow W_{*}(\Phi)=\sum_{n=0}^{\infty} \frac{1}{n !} W^{(n)} \Phi * \Phi * \stackrel{(n)}{*} * \Phi
$$

where the superfield $\Phi$ has the component expansion (2.3),

$$
\Phi(t, \theta, \bar{\theta})=\phi(t)+\theta \psi(t)+\bar{\psi}(t) \bar{\theta}+\theta \bar{\theta} F(t)
$$

In terms of the $(\tau, \theta, \bar{\theta})$ coordinates, the superfield $\Phi$ is real, that is, $\phi^{\dagger}(\tau)=\phi(\tau), \psi^{i}(\tau)=$ $\bar{\psi}(\tau)$ and $F^{\dagger}(\tau)=F(\tau)$. However, the change of variables $\tau \rightarrow t$ complexifies the temporal coordinate and then, the reality relations for the component fields are lost.

In the two-dimensional NAC $\mathcal{N}=2$ sigma model context Alvarez-Gaumé and VázquezMozo found a remarkable non-perturbative formula for the deformed holomorphic superpotential [23]-[25]. Now, this formula can be also applied in this $d=1$ case and allows us to express the higher component of the superpotential as

$$
\int d \theta d \bar{\theta} W_{*}(\Phi)=F \frac{\partial}{\partial \phi} \int_{-\frac{1}{2}}^{+\frac{1}{2}} d \xi W(\phi+\xi c F)-\bar{\psi} \psi \frac{\partial^{2}}{\partial \phi^{2}} \int_{-\frac{1}{2}}^{+\frac{1}{2}} d \xi W(\phi+\xi c F)
$$

with

$$
c=\sqrt{C \bar{C}-\widehat{C} \bar{C}}
$$

As noted in [23]-[25], this deformation corresponds, physically, to a smearing of the target space coordinates. According to this, an effective superpotential $\bar{W}$ can be obtained by averaging its undeformed value between $\phi-c F$ and $\phi+c F$, that is

$$
\tilde{W}(\phi, F)=\int_{-\frac{1}{2}}^{+\frac{1}{2}} d \xi W(\phi+\xi c F) .
$$

So, written in components, the deformed action for Quantum Mechanics takes the form

$$
S=\int d t\left[-\left(i \frac{d \phi}{d t}+\frac{\partial \hat{W}}{\partial \phi}\right) F+\frac{1}{2} F^{2}+\bar{\psi}\left(i \frac{d}{d t}+\frac{\partial^{2} \tilde{W}}{\partial \phi^{2}}\right) \psi\right]
$$

In order to study classical aspects of this deformed theory let us use the usual definition for the momenta, Hamiltonian and Poisson brackets

$$
\Pi^{i}=\frac{\partial_{r} \mathcal{L}}{\partial \dot{\Theta}_{i}}, \quad \mathcal{H}=\Pi^{i} \dot{\Theta}_{i}-\mathcal{L}, \quad\{X, Y\}_{P}=\frac{\partial_{r} X}{\partial \Theta_{i}} \frac{\partial_{l} Y}{\partial \Pi^{i}}-(-1)^{\mathrm{XY}} \frac{\partial_{r} Y}{\partial \Theta_{i}} \frac{\partial_{l} X}{\partial \Pi^{i}},
$$

where the subscripts $r$ and $l$ denotes right and left derivatives, respectively. Hence, with $\Theta=(\phi, \psi)$, the conjugate momenta are $\Pi=(-i F, i \bar{\psi})$ and the Hamiltonian associated with the deformed action (3.7) is

$$
\mathcal{H}=-i F \dot{\phi}+i \bar{\psi} \dot{\psi}-\mathcal{L}=\frac{\partial \bar{W}}{\partial \phi} F-\frac{1}{2} F^{2}-\bar{\psi} \frac{\partial^{2} \bar{W}}{\partial \phi^{2}} \psi
$$


One can easily check that the action (3.7) is still invariant under the supersymmetry generated by $Q$

$$
\delta_{Q} \phi=\psi, \quad \delta_{Q} \psi=0, \quad \delta_{Q} \bar{\psi}=F, \quad \delta_{Q} F=0,
$$

where the variations for the fields are obtained from

$$
\delta_{Q} \Phi=Q * \Phi=\delta_{Q} \phi-\theta \delta_{Q} \psi+\delta_{Q} \bar{\psi} \bar{\theta}+\theta \bar{\theta} \delta_{Q} F
$$

On the other hand, we have seen that the differential operator $\bar{Q}$ as defined in (2.14) and satisfying $\{\bar{Q}, \bar{Q}\} \neq 0$, cannot be used as a generator of SUSY transformations. Besides, the $Q$-variation of the component fields corresponding to the undeformed case are no more a symmetry of the deformed theory. That is, given the variations

$$
\delta_{\bar{Q}_{c=0}} \phi=\bar{\psi}, \quad \delta_{\bar{Q}_{c=0}} \psi=2 i \dot{\phi}-F, \quad \delta_{\bar{Q}_{c=0}} \bar{\psi}=0, \quad \delta_{\bar{Q}_{c=0}} F=2 i \dot{\bar{\psi}},
$$

the Lagrangian does not remain invariant but changes according to

$$
\delta_{\bar{Q}_{c=0}} \mathcal{L}=2 i \bar{\psi} \frac{\partial^{2} \tilde{W}}{\partial F \partial \phi} \dot{F}-2 i \dot{\bar{\psi}} \frac{\partial^{2} \tilde{W}}{\partial F \partial \phi} F-2 i \bar{\psi} \dot{\psi} \frac{\partial^{3} \tilde{W}}{\partial F \partial \phi^{2}} \psi
$$

Then, only for $c=0$ (the undeformed case) $\delta_{Q_{c=0}} \mathcal{L}$ vanishes. This result was to be expected from the form of the deformed algebra of the supercharge (2.10) which, after taking $b=0, \bar{b}=2$, is broken in the $\bar{Q}$ sector. This is similar to what happens in NAC field theories where at least half of the original supersymmetry is broken due to the deformation.

Coming back to the supersymmetry that is preserved, we can give an expression of the supercharge $Q$ in terms of the fields following the Noether prescription,

$$
\mathcal{Q}=-\sum_{\chi} \frac{\partial_{r} \mathcal{L}}{\partial \dot{\chi}} \delta_{Q} \chi=i \psi F,
$$

(with $\chi$ we denote the component fields in the theory). One can see that $\mathcal{Q}$ satisfies

$$
\{\mathcal{Q}, \mathcal{Q}\}_{P}=0, \quad\{\mathcal{Q}, \chi\}_{P}=\delta_{Q} \chi
$$

for the variations (3.10). One can then check that the Hamiltonian is $\mathcal{Q}$-exact since

$$
\mathcal{H}=\left\{\mathcal{Q}, \frac{1}{2} \bar{\psi}\left(-F+2 \frac{\partial \tilde{W}}{\partial \phi}\right)\right\}_{P}
$$

This expression suggests the existence of a second charge $\mathcal{Q}$ so that, as in the undeformed case, the deformed Hamiltonian could be written as

$$
\mathcal{H}=\frac{i}{2}\{\mathcal{Q}, \overline{\mathcal{Q}}\}_{P}
$$

This can be achieved by defining the charge $\overline{\mathcal{Q}}$ so that, in terms of component fields, it takes the form

$$
\overline{\mathcal{Q}}=i \bar{\psi}\left(F-2 \frac{\partial \tilde{W}}{\partial \phi}\right)
$$


Surprisingly, the charge $\mathcal{Q}$ is conserved,

$$
\{\mathcal{H}, \overline{\mathcal{Q}}\}_{P}=0
$$

and it is also nilpotent,

$$
\{\mathcal{Q}, \mathcal{Q}\}_{P}=0
$$

We conclude that, at least classically, the deformed theory has as many supercharges as the undeformed one. Acting on the fields, the second charge gives the transformations

$$
\begin{array}{ll}
\{\overline{\mathcal{Q}}, \phi\}_{P}=\bar{\psi}-2 \bar{\psi} \frac{\partial^{2} \tilde{W}}{\partial \phi \partial F}, & \{\overline{\mathcal{Q}}, \bar{\psi}\}_{P}=0, \\
\{\overline{\mathcal{Q}}, \psi\}_{P}=F-2 \frac{\partial \tilde{W}}{\partial \phi}, & \{\overline{\mathcal{Q}}, F\}_{P}=2 \bar{\psi} \frac{\partial^{2} \bar{W}}{\partial \phi^{2}} .
\end{array}
$$

Note that in the $c \rightarrow 0$ limit $\overline{\mathcal{Q}} \rightarrow \bar{Q}_{c=0}$, as one can verify by comparing transformations (3.12) and (3.21) and using component fields equations of motion. It is important to stress that, in contrast with the case of the transformations (3.12), which gives a linear realization of the supersymmetry algebra in the undeformed theory, $\overline{\mathcal{Q}}$ provides, together with $\mathcal{Q}$, a non-linear realization defined by eqs. (3.15),(3.21).

\section{Quantum supersymmetries}

To study the quantum theory, one should replace the Poisson bracket by commutators, $\{,\}_{P} \rightarrow-i[$,$] and write the canonical commutation relations$

$$
\left[\phi, \Pi_{\phi}\right]=i, \quad\{\psi, i \bar{\psi}\}=1 .
$$

to describe the Hilbert space. The first relation can be represented in the standard way in terms of wavefunctions in $L^{2}(\mathbb{R})$. To represent the second one, which corresponds to a Clifford algebra, we can use the $2 \times 2$ matrices,

$$
\psi=\left(\begin{array}{cc}
0 & 1 \\
0 & 0
\end{array}\right), \quad i \bar{\psi}=\left(\begin{array}{cc}
0 & 0 \\
1 & 0
\end{array}\right) .
$$

In order to study symmetries at the quantum level it will be more convenient to work with a new charge

$$
\mathcal{Q}_{+}=\underline{\mathcal{Q}}+\underline{\mathcal{Q}}
$$

(or equivalently with $\mathcal{Q}_{-}=i\left(\mathcal{Q}_{-} \mathcal{Q}\right)$ ) which satisfies $\mathcal{H}=\left(\mathcal{Q}_{+}\right)^{2}$. Note that this relation imposes a constraint to the possible orderings of $\mathcal{H}$ and $\mathcal{Q}_{+}$.

In order to determine whether the $\mathcal{Q}$-supersymmetries are spontaneously broken in the deformed theory, one can analyze the Witten index. Since the Hamiltonian is the square of $\mathcal{Q}_{+}$, then $\left[\mathcal{H}, \mathcal{Q}_{1}\right]=0$. Thus, except for states annihilated by $\mathcal{Q}_{1}$, a boson/fermion pairing of states with the same energy takes place and the Witten index results

$$
\mathcal{W}=\operatorname{Tr}\left[(-1)^{F} e^{-i \mathcal{H}}\right]=\left\{\sharp \text { bosonic } \Upsilon_{+}^{\text {closed states }}\right\}-\left\{\sharp \text { fermionic } \Upsilon_{+}^{\text {cllosed states }}\right\}
$$

where $\Upsilon_{+}^{\text {closed states }}$ are those states which are annihilated by $\mathcal{Q}_{+}$. 
Were $\mathcal{Q}_{+}$an Hermitian operator, the following identity would be valid

$$
\left.\langle\chi|\mathcal{H}| \chi\rangle=\left|\mathcal{Q}_{+}\right| \chi\right\rangle\left.\right|^{2}
$$

and then states annihilated by $\mathcal{Q}_{+}$would be vacuum states. As a consequence, a non vanishing Witten index would imply that the $\mathcal{Q}_{+}$-symmetry is not broken. This is in fact what happens for supersymmetric theories in undeformed superspace. Now, when the deformation is present the situation radically changes: as it happens for non(anti)commutative field theories, the action, and hence the Hamiltonian, are in general complex. Then, according to $(3.17), \mathcal{\mathcal { Q }}$ is not the hermitian conjugate of $\mathcal{Q}$, this leading in general to non-hermitian charges $\mathcal{Q}_{ \pm}$.

Now, it has been proved [26] that the Witten index can be obtained from the partition function $Z$ in Euclidean time through standard manipulations,

$$
\mathcal{W}=\int_{\mathrm{PBC}} \mathcal{D} \Theta \mathcal{D} \Pi \exp \left(-\int_{0}^{\beta} d t\left(\Pi^{i} \dot{\Theta}_{i}-\mathcal{H}\right)\right),
$$

with $\Theta=(\phi, \psi), \Pi=(i F,-i \bar{\psi})$. Canonical variables in the Hamiltonian operator should be orderer so that they satisfy $\mathcal{H}=e^{i p_{\phi} \phi}\left\langle p_{\phi}|\mathcal{H}| \phi\right\rangle$ and the functional integral is taken over fields satisfying periodic boundary conditions: $\Theta(t+\beta)=\Theta(t)$. With this representation for the Witten index, its calculation can be easily performed since the deformed partition function coincides with the undeformed one when the $\mathcal{Q}$-symmetry is not broken in the undeformed $(c=0)$ theory. To see this we should first note that the deformed action is $\mathcal{Q}$-exact, that is,

$$
S=\int d t\left\{\mathcal{Q}, \bar{\psi}\left(\frac{d \phi}{d t}+\frac{\partial \bar{W}}{\partial \phi}-\frac{i}{2} F\right)\right\}_{P}=S(c=0)+\int d t\left\{\mathcal{Q}, \bar{\psi} \frac{\partial}{\partial \phi}(\tilde{W}-W)\right\}_{P}
$$

As a result, we can express the partition function as

$$
\begin{aligned}
& Z=\int \mathcal{D} \Phi \exp (-S)=\int \mathcal{D} \Phi \exp \left(-S(c=0)-\int d t\left\{\mathcal{Q}, \bar{\psi} \frac{\partial}{\partial \phi}(\tilde{W}-W)\right\}_{P}(t)\right) \\
&=Z(c=0)+ \sum_{n>0}(-1)^{n} \int d t_{1} \ldots \int d t_{n} \times \\
&\left\langle\left\{\mathcal{Q}_{c=0}, \bar{\psi} \frac{\partial}{\partial \phi}(\tilde{W}-W)\right\}\left(t_{1}\right) \ldots\left\{\mathcal{Q}_{c=0}, \bar{\psi} \frac{\partial}{\partial \phi}(\tilde{W}-W)\right\}\left(t_{n}\right)\right\rangle_{c=0}
\end{aligned}
$$

where \langle\rangle$_{c=0}$ denote the expectation value corresponding to the undeformed case. Note that the replacement $\{\mathcal{Q},\} \rightarrow\left\{\mathcal{Q}_{c=0},\right\}$ can be done since the $\mathcal{Q}$-transformations of the field components are not affected by the deformation. If $\mathcal{Q}_{c=0}$-symmetry is not broken in the undeformed case, expectation values of $\mathcal{Q}_{c=0}$-exact operators vanish. Thus we get that $Z=Z(c=0)$ and, due to relation $(4.6), \mathcal{W}=\mathcal{W}(c=0)$ when the undeformed theory is supersymmetric.

We can summarize the latter results as follows,

$$
\begin{aligned}
& \mathcal{W}=\left\{\sharp \text { bosonic } \Upsilon_{+}^{\text {closed states }}\right\}-\left\{\sharp \text { fermionic } \Upsilon_{+}^{\text {closed states }}\right\}= \\
& \left\{\begin{array}{c}
-1 \text { for } W(\phi) \rightarrow a \phi^{2 n} \text { as } \phi \rightarrow \pm \infty, a>0 \\
1 \quad \text { for } W(\phi) \rightarrow-a \phi^{2 n} \text { as } \phi- \pm \infty \\
\text { unknown in other case. }
\end{array}\right.
\end{aligned}
$$


Taking vacuum states as those for which the v.e.v. of $\mathcal{H}$ vanishes, the states annihilated by $\mathcal{Q}_{+}$are vacuum states. Then, those cases in which $\mathcal{W} \neq 0$ correspond to theories with unbroken supersymmetry.

\section{The $(2,2,0)$ supermultiplet}

When one formulates the deformed theory in terms of the $(\mathbf{2}, \mathbf{2}, 0)$ supermultiplet, that is, by using complex chiral superfields instead of real ones, auxiliary fields are not introduced. It could then be promising to analyze this case since it was the presence of the auxiliary field which prevented a complete answer to the SUSY symmetry breaking in the case of the $(1,2,1)$ multiplet.

In the chiral base, a chiral superfield $\Psi$ and an antichiral superfield $\Psi$ have the component expansions

$$
\begin{aligned}
\Psi(t, \theta) & =z(t)+\theta \chi(t) \\
\bar{\Psi}(t-2 i \theta \bar{\theta}, \bar{\theta}) & =\bar{z}(t-2 i \theta \bar{\theta})+\bar{\chi}(t) \bar{\theta}=\bar{z}(t)+\bar{\chi}(t) \bar{\theta}-2 i \theta \bar{\theta} \dot{\bar{z}}(t)
\end{aligned}
$$

In terms of $(\tau, \theta, \bar{\theta})$ coordinates, the component fields satisfy $z^{\dagger}(\tau)=\bar{z}(\tau)$ and $\chi^{\dagger}(\tau)=\bar{\chi}(\tau)$. Now, in order to use these supermultiplets one needs the Moyal-Weyl product to preserve the superfields chirality condition. With the generic deformation (2.2), the products of chiral and antichiral superfields take the form

$$
\begin{aligned}
& \Psi_{1} * \Psi_{2}=\Psi_{1} \Psi_{2}-\frac{C}{2} \chi_{1} \chi_{2} \\
& \Psi_{1} * \Psi_{2}=\bar{\Psi}_{1} \Psi_{2}-\frac{C}{2} \bar{\chi} \bar{\chi}_{1}+c^{2} \dot{\bar{z}}_{1} \dot{\bar{z}}_{2}+i C \theta\left(\dot{\bar{z}}_{1} \bar{\chi}_{2}-\dot{\hat{z}}_{2} \bar{\chi}_{1}\right)+i \widehat{C}\left(\dot{\bar{z}}_{1} \bar{\chi}_{2}-\dot{\bar{z}}_{2} \bar{\chi}_{1}\right) \bar{\theta}
\end{aligned}
$$

One can see that a product of chiral superfield is still chiral. However, in order to preserve the antichirality condition, the deformation has to satisfy $\bar{C}=c^{2}=0$. Then, only the $\theta$ algebra gets deformed.

Let us consider a theory with the kinetic energy canonically normalized and a real Kähler prepotential $K(z, \bar{z})$. The deformed action is

$$
\left.S=\int d t d \theta d \bar{\theta}\left[\frac{1}{4}(D * \Psi) *(\bar{D} * \bar{\Psi})+K_{*}(\Psi, \bar{\Psi})\right]\right)
$$

with the deformed prepotential defined as

$$
K_{*}(\Psi, \bar{\Psi})=\sum_{n, m=0}^{\infty} \frac{1}{(n+m) !} \frac{\partial^{n+m} K}{\partial z^{n} \partial \bar{z}^{m}}[\Psi * !(n) * \Psi * \bar{\Psi} *(m) * \bar{\Psi}]
$$

where the square brackets [...] means all possible permutations of the superfields.

A non-perturbative expression for the deformed Kähler prepotential can be obtained using a generalization of ec. (3.4) for several superfields [23]-[25]. However, as in the case of one superfield, the deformation of the prepotential is controlled by the parameter $c^{2}$, which in this case is zero, and then the prepotential does not receive corrections. As the kinetic term neither is affected by the deformation, the action (5.3) results undeformed. 


\section{Summary and discussion}

We have analyzed in this work NAC deformations of the $d=1 \mathcal{N}=2$ superspace and studied how a Quantum Mechanics model can be defined on it. In particular, the emphasis was put on the determination of how many supercharges were broken due to the NAC deformation. Starting from a general deformation of the superspace fermionic coordinates and working in the chiral base, we found that one half of the original supersymmetry algebra (corresponding to the supercharge $Q$ ) can be preserved, the same as it happens in NAC field theories.

By using a Moyal-Weyl product to realize the deformation, we defined a deformed $\mathcal{N}=2$ SUSY theory in terms of the $(\mathbf{1}, \mathbf{2}, \mathbf{1})$ supermultiplet for an arbitrary superpotential. To do this we applied a remarkable result [23]-[25] for the deformed two dimensional sigma model which allows to obtain a closed expression for the deformed superpotential. In this way we found that the resulting Lagrangian takes the same form as the undeformed one but with a superpotential which is the average of its undeformed value in the interval $(\phi-c F / 2, \phi+c F / 2)$, with $c$ related to the deformation as given by formula (3.5). As a consequence, the NAC deformed superpotential depends not only on $\phi$ but also on the auxiliary field $F$.

Concerning the supersymmetries of the deformed model, an expression in terms of the component fields for the surviving charge $\mathcal{Q}$ was easily found by using its differential operator representation (2.14). The fact that the Hamiltonian was still $\mathcal{Q}$-exact made natural to propose an ansatz for a second supercharge $\overline{\mathcal{Q}}$. This charge is found to be conserved and together with $\mathcal{Q}$, defines a nonlinear realization of the supersymetry algebra:

$$
\{\mathcal{Q}, \mathcal{Q}\}_{P}=-2 i \mathcal{H}, \quad\{\mathcal{Q}, \mathcal{Q}\}_{P}=0, \quad\{\mathcal{Q}, \overline{\mathcal{Q}}\}_{P}=0
$$

As a result, we have the unexpected result that the deformed model has as many (conserved) supercharges as the undeformed one.

We analyzed the conservation of these supercharges in the quantum theory and whether they were or not spontaneously broken. The fact that the deformed Hamiltonian is, in general, non hermitian leads to non-hermiticity of supercharges. However, we found that, whenever the undeformed theory is supersymetric, the Witten index, defined as a pathintegral, does not change with the deformation. This result allowed to determine the difference between the number of boson and fermion states annihilated by the supercharge and then, to know when the supercharge is not spontaneously broken.

Finally, we also formulated the deformed theory in terms of the $(\mathbf{2}, \mathbf{2}, \mathbf{0})$ supermultiplet, that is, by using complex chiral superfields instead of real ones. In this case, requiring the Moyal-Weyl product to preserve the chirality condition imposes constraints on the class of deformations. Now these constraints turn out to be too restrictive so that finally the resulting deformed action coincides with the undeformed one.

Our work suggests that NAC quantum field theories could have more supersymmetries than those one naively expect in view of the deformation. It would be very interesting to gain a deeper insight in this direction. 
Non(anti)commutative Quantum Mechanics is also a very interesting topic for further analysis in connection with the no-linear sigma model. It is known that the addition of more "superspace structure" by means of NAC deformations leads to new deformations in complex geometry, whose geometrical significance is yet to be understood [27]. The study of NAC deformed one dimensional no-linear sigma model can shed some light on this issue.

Finally, as it happens with Noncommutative QM, non(anti) commutative QM could have applications in condensed matter problems. For instance, it has been shown that, in supermatrix models, fuzzy superspheres arise as classical solutions, and their fluctuations yield to NAC field theories [28]. Some interesting relations between lowest Landau level (LLL) physics and NAC geometry have also been reported [29, 30]. With these recent developments, the supersymmetric quantum Hall systems might be the simplest "physical" set-up of NAC geometry.

\section{Acknowledgments}

F.A.S. wishes to thank L. Cugliandolo, E. Fradkin, E. Moreno and J. Kurchan for their comments and patience the many times they had to hear about the ideas behind this work. L.A. is supported by CONICET. This work is partially supported by UNLP, CICBA, CONICET (PIP 6160) and ANPCYT.

\section{References}

[1] J.H. Schwarz and P. Van Nieuwenhuizen, Speculations concerning a fermionic substructure of space-time, Lett. Nuovo Cim. 34 (1982) 21.

[2] S. Ferrara and M.A. Lledo, Some aspects of deformations of supersymmetric field theories, JHEP 05 (2000) 008 [hep-th/0002084].

[3] D. Klemm, S. Penati and L. Tamassia, Non(anti)commutative superspace, Class, and Quant. Grav, 20 (2003) 2905 [hep-th/0104190].

[4] H. Ooguri and C. Vafa, The c-deformation of gluino and non-planar diagrams, Adv. Theor. Math. Phys. 7 (2003) 53 [hep-th/0302109].

[5] H. Ooguri and C. Vafa, Gravity induced c-deformation, Adv. Theor. Math. Phys. 7 (2004) 405 [hep-th/0303063].

[6] N. Seiberg, Noncommutative superspace, $N=1 / 2$ supersymmetry, field theory and string theory, JHEP 06 (2003) 010 [hep-th/0305248].

[7] N. Berkovits and N. Seiberg, Superstrings in graviphoton background and $N=1 / 2+3 / 2$ supersymmetry, JHEP 07 (2003) 010 [hep-th/0306226].

[8] J. de Boer, P.A. Grassi and P. van Nieuwenhuizen, Non-commutative superspace from string theory, Phys. Lett. B 574 (2003) 98 [hep-th/0302078].

[9] M. Billo, M. Frau, I. Pesando and A. Lerda, $N=1 / 2$ gauge theory and its instanton moduli space from open strings in RR background, JHEP 05 (2004) 023 [hep-th/0402160].

[10] P.A. Grassi and L. Tamassia, Vertex operators for closed superstrings, JHEP 07 (2004) 071 [hep-th/0405072]. 
[11] M. Billo, M. Frau, F. Lonegro and A. Lerda, $N=1 / 2$ quiver gauge theories from open strings with RR fluxes, JHEP 05 (2005) 047 [hep-th/0502084].

[12] L. Tamassia, Noncommutative supersymmetric/integrable models and string theory, hep-th/0506064.

[13] P.A. Grassi, $N=2$ superparticles, $R R$ fields and noncommutative structures of (super)-spacetime, hep-th/0511015.

[14] L.G. Aldrovandi, F.A. Schaposnik and G.A. Silva, A coordinate-dependent superspace deformation from string theory, JHEP 03 (2006) 038 [hep-th/0602108].

[15] A. Imaanpur, Comments on gluino condensates in $N=1 / 2 S Y M$ theory, JHEP 12 (2003) 009 [hep-th/0311137].

[16] A. Gorsky and M. Shifman, Spectral degeneracy in supersymmetric gluodynamics and one-flavor QCD related to $N=1 / 2$ SUSY, Phys. Rev. D 71 (2005) 025009 [hep-th/0410099].

[17] C.-S. Chu and T. Inami, Konishi anomaly and central extension in $N=1 / 2$ supersymmetry, Nucl. Phys. B 725 (2005) 327 [hep-th/0505141].

[18] L.G. Aldrovandi, D.H. Correa, F.A. Schaposnik and G.A. Silva, BPS analysis of gauge field Higgs models in non- anticommutative superspace, Phys. Rev. D 71 (2005) 025015 [hep-th/0410256];

L.G. Aldrovandi, F.A. Schaposnik and G.A. Silva, Non(anti)commutative superspace with coordinate-dependent deformation, Phys. Rev. D 72 (2005) 045005 [hep-th/0505217].

[19] E. Witten, Dynamical breaking of supersymmetry, Nucl. Phys. B 188 (1981) 513.

[20] E. Witten, Constraints on supersymmetry breaking, Nucl. Phys. B 202 (1982) 253.

[21] L. Alvarez-Gaume, Supersymmetry and the Atiyah-Singer index theorem, Commun. Math. Phys. 90 (1983) 161.

[22] D. Friedan and P. Windey, Supersymmetric derivation of the Atiyah-Singer index and the chiral anomaly, Nucl. Phys. B 235 (1984) 395.

[23] T. Hatanaka, S.V. Ketov, Y. Kobayashi and S. Sasaki, Non-anti-commutative deformation of effective potentials in supersymmetric gauge theories, Nucl. Phys. B 716 (2005) 88 [hep-th/0502026].

[24] L. Alvarez-Gaumé and M.A. Vazquez-Mozo, On nonanticommutative $N=2$ sigma-models in two dimensions, JHEP 04 (2005) 007 [hep-th/0503016].

[25] T. Hatanaka, S.V. Ketov, Y. Kobayashi and S. Sasaki, $N=1 / 2$ supersymmetric four-dimensional non-linear sigma-models from non-anti-commutative superspace, Nucl. Phys. B 726 (2005) 481 [hep-th/0506071].

[26] S. Cecotti and L. Girardello, Functional measure, topology and dynamical supersymmetry breaking, Phys. Lett. B 110 (1982) 39.

[27] S.V. Ketov, Non-anti-commutative deformation of complex geometry, hep-th/0602066.

[28] S. Iso and H. Umetsu, Gauge theory on noncommutative supersphere from supermatrix model, Phys. Rev. D 69 (2004) 105003 [hep-th/0311005].

[29] M. Hatsuda, S. Iso and H. Umetsu, Noncommutative superspace, supermatrix and lowest Landau level, Nucl. Phys. B 671 (2003) 217 [hep-th/0306251]. 
[30] E. Ivanov, L. Mezincescu and P.K. Townsend, Fuzzy $C P(n \mid m)$ as a quantum superspace, hep-th/0311159. 